\title{
Exercise Is Associated with a Reduction in Gestational Diabetes Mellitus
}

\author{
YAIZA CORDERO ${ }^{1}$, MICHELLE F. MOTTOLA ${ }^{2}$, JUANA VARGAS $^{3}$, MAITE BLANCO $^{4}$, and RUBÉN BARAKAT ${ }^{5}$ \\ ${ }^{1}$ Physical Activity and Sport Science Faculty, Catholic University of Murcia (UCAM), Murcia, SPAIN; ${ }^{2}$ R. Samuel McLaughlin \\ Foundation Exercise and Pregnancy Lab, School of Kinesiology, The University of Western Ontario, London, Ontario, \\ CANADA; ${ }^{3}$ Gynecology and Obstetric Department of Puerta de Hierro University Hospital, Madrid, SPAIN; ${ }^{4}$ Gynecology \\ and Obstetric Department of Torrelodones University Hospital, Madrid, SPAIN; and ${ }^{5}$ Physical Activity and Sport Science \\ Faculty (INEF), Technical University of Madrid, Madrid, SPAIN
}

\begin{abstract}
CORDERO, Y., M. F. MOTTOLA, J. VARGAS, M. BLANCO, and R. BARAKAT. Exercise Is Associated with a Reduction in Gestational Diabetes Mellitus. Med. Sci. Sports Exerc., Vol. 47, No. 7, pp. 1328-1333, 2015. Purpose: The objective of this study is to assess the effectiveness of a maternal exercise program (land/aquatic activities, both aerobic and muscular conditioning) in preventing gestational diabetes mellitus (GDM). Methods: Three hundred and forty-two pregnant women from Spain (age, $33.24 \pm 4.3$ yr) without obstetric contraindications were recruited for a clinical randomized controlled trial. The intervention group (IG, $n=101$ ) exercised for 60 and $50 \mathrm{~min}$ on land and in water, respectively, three times per week. The control group $(n=156)$ received usual standard care. Results: The prevalence of GDM was reduced in the IG group (IG, $1 \%, n=1$, vs control group, $8.8 \%, n=13\left(\chi^{2}{ }_{1}=6.84, P=0.009\right)$ ) with a significant risk estimate (odds ratio $=0.103 ; 95 \%$ confidence interval, $0.013-0.803$ ). Conclusion: The exercise program performed during pregnancy reduced the prevalence of GDM by preserving glucose tolerance. Key Words: PHYSICAL ACTIVITY, PREGNANCY, GLUCOSE TOLERANCE, BMI, EXCESSIVE MATERNAL WEIGHT GAIN
\end{abstract}

$\mathrm{D}$ iabetes is mostly attributed to obesity and physical inactivity, and in recent years, intrauterine exposure has been added as a contributing factor. If maternal glucose intolerance is present, the offspring may be predisposed to future disease risk (30). This vicious circle can influence and perpetuate the incidence and prevalence of glucose intolerance (30). Women with gestational diabetes mellitus (GDM) have an increased risk of developing type 2 diabetes (3).

The prevalence of GDM varies around the world, even between racial and ethnic groups in the same country. Currently, there is no consensus about the diagnostic criteria, making it difficult to obtain accurate estimates of prevalence between countries (from $1 \%$ to $16 \%$ depending on the population studied, the selection of protocols, and diagnostic criteria used) $(27,30)$. The increased trend in the prevalence across the globe and the risks for public health cannot be ignored. In Spain, according to the diagnostic criteria of the National Diabetes Data Group, there is a prevalence of $8.8 \%$

\footnotetext{
Address for correspondence: Yaiza Cordero, Ph.D., Physical Activity and Sport Science Faculty, Catholic University of Murcia, Campus de los Jerónimos, s/n, 30107, Guadalupe-Murcia, Spain; E-mail: yaiza.cordero@gmail.com. Submitted for publication August 2014.

Accepted for publication October 2014.

0195-9131/15/4707-1328/0

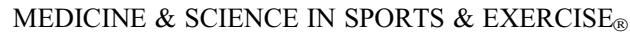
Copyright $(2014$ by the American College of Sports Medicine

DOI: 10.1249/MSS.0000000000000547
}

for GDM (28). Exercise is an essential element for glucose metabolic control (25) and may prevent GDM.

Exercise increases the rate of glucose uptake in skeletal muscle, a process that is regulated by the GLUT4 transporter (37). This explains the relationship observed between physical exercise and improvement in maternal glucose homeostasis and insulin sensitivity $(14,25,32)$. Similarly, exercise during pregnancy is associated with better glucose tolerance (25). Studies report a lower administration of insulin in women with GDM who performed physical exercise versus those who remained inactive $(10,23)$. There has also been a positive link between the initiation of exercise in women who were inactive the year before pregnancy and during pregnancy in preventing GDM (34). However, the type, duration, and intensity of exercise during pregnancy to prevent GDM have yet to be defined (26).

The purpose of the current study was to examine the efficacy of a physical exercise program during pregnancy to prevent GDM. It was hypothesized that a combined exercise program on land and water, employing aerobic and muscle toning activities, is an effective tool for the prevention of GDM.

\section{METHODS}

A randomized clinical trial was designed, controlled, and unmasked. It included two study groups: intervention group (IG) that followed a program of physical exercise and a control group $(\mathrm{CG})$ that remained inactive. (This study was registered at www.clinicaltrials.gov, identifier: NCT01790412.) 
To obtain a minimum power of $80 \%$ in the analysis of the relationship between the introduction of exercise and the appearance of GDM, a medium effect size was set with a $95 \%$ level of confidence $(\alpha=0.05)$. The size of the sample was calculated as a minimum of 87 subjects per group (9).

\section{Participants}

Pregnant women living in the health care area of Hospital Puerta de Hierro, Madrid, Spain (age, $33.24 \pm 4.3$ yr), were recruited at 10 to $12 \mathrm{wk}$ of gestation from their first trimester ultrasound appointment. None of the women experienced medical obstetric contraindications (1), and all of them had medical clearance for physical exercise. They were not previously familiar with the types of exercise included in the program. A minimum of $80 \%$ adherence to the exercise classes was required for women assigned to the IG.

\section{Physical Activity Program}

The physical activity program was carried out between weeks 10 and 14 to the end of the third trimester. Women in the IG exercised for 50- to 60-min sessions, three sessions per week, two on land (gym hall) and one as an aquaticwater-based activity (small and large pool tanks). The exercise intensity was set through Borg's scale-between 6 (without effort) and 20 (maximum effort) (4). For the exercise sessions, level 12-14 was maintained-i.e., somewhat strong (2). In addition, maternal HR was assessed by using an HR monitor (Accurex Plus, Polar Electro OY, Finland) and exercise intensity was modulated in order not to surpass $60 \%$ of the calculated HR reserve [(220 - age) - (resting HR) $\times 60 \%$ ] + resting HR (17). To maximize patient safety and adherence to the training program and its efficacy, all of the sessions were supervised by a qualified fitness specialist (working with groups of 10-12 women) with the assistance of an obstetrician.

On-land session. The land sessions were divided into specific parts: phase 1 consisted of activation and physical and psychological preparation. These were composed of various displacements following distinct forms, locomotive games, articular movement, and light stretches. After this, lowimpact aerobic choreography was followed (aerobics, fitness, modern dance, Latin dance, cardio boxing, rhythm, and percussion). In the following phase, body toning was achieved through muscular exercise/work directed at almost all muscle groups (abdominal exercises were avoided). A program of two series of 15 repetitions was completed for each of different muscle groups: biceps and triceps exercises with dumbbells weighing $2 \mathrm{~kg}$ and quadriceps stimulation using lunges and gluteal work on all fours using their body weight.

Exercises that involved the Valsalva maneuver, extreme stretching, joint overextension, ballistic movements, and jumping were specifically avoided. Furthermore, the exercises were performed in the supine position for no longer than $2 \mathrm{~min}$.

As to the pelvic floor exercise block, an initial identification and awareness phase took place (in the first month) followed by work focusing on slow and fast contractions in the affected zones, in different positions, and gradually increasing the volume of work, culminating at approximately 100 repetitions per session. In the final cool down phase, work was focused on the flexibility of the muscular groups most affected by pregnancy: the lumbar area, gluteus, psoas, calf muscles, neck, and shoulders. This block included additional relaxation and visualization exercises, self-massage, and pair massage.

The sessions were accompanied by music with a range of 110 to $168 \mathrm{bpm}$ in the active phase of each session, while a range of 66 to $76 \mathrm{bpm}$ was used in the pelvic floor and cool down phase.

Materials like foam rubber balls of differing sizes, elastic bands, Pilates rings, 2-kg dumbbells, 1-kg dumbbells, 45-cm Swiss balls (fitness balls), mats of different sizes and thicknesses, and rolled or wedged pillows were used.

Aquatic activities. Aquatic sessions included an initial activation (various displacements and smooth movements of the upper and lower body). The central part of the work was divided between (a) displacements while swimming (except butterfly style) and (b) strength exercises and aquatic activities (propulsion exercises). The final part consisted of flexibility exercises, relaxation, and breathing in the small pool tank.

Aquatic materials like foam rubber balls of differing sizes, swimming accessories such as floats, pull buoys (buoyancy aiding devices), water noodles, armbands, and rubber rings were used. Swimming mitts were also provided for muscle conditioning and floating weights for resistance to movement. Water temperature was $28.5^{\circ} \mathrm{C}-29^{\circ} \mathrm{C}$.

Timing of sessions. The land aerobic sessions consisted of a gradual warm-up (10 $\mathrm{min})$, aerobic choreography (20 $\mathrm{min})$, resistance exercises $(12 \mathrm{~min})$, pelvic floor exercises $(10 \mathrm{~min})$ followed by stretching $(8 \mathrm{~min})$.

The aquatic activities included a gradual warm-up (10 min), a core session of swimming laps, step climbs, lunges and strength exercises in the water (30 $\mathrm{min})$, and stretching (10 min). All sessions were supervised by a qualified fitness specialist (working with groups of 10-12 women).

\section{Variables of the Study}

The primary outcome. The primary outcome was the diagnosis of GDM. The National Diabetes Data Group criteria were followed and included a 50-g maternal glucose screen (MGS) at 24-28 wk of gestation, which was to determine plasma glucose $1 \mathrm{~h}$ after a 50-g load of glucose administered orally. The screen test was considered positive when the values were equal or greater than $140 \mathrm{mg} \cdot \mathrm{dL}^{-1}$ $\left(7.8 \mathrm{mmol} \cdot \mathrm{L}^{-1}\right)$. If the screen test was positive, the women were required to have a fasted oral glucose tolerance test (OGTT) before $30 \mathrm{wk}$ of gestation with data taken from medical records: $100 \mathrm{~g}$ of glucose load with blood samples taken fasted and at 1,2 , and $3 \mathrm{~h}$ postglucose ingestion. Positive results were fasting glucose equal to or greater than $105 \mathrm{mg} \cdot \mathrm{dL}^{-1}\left(5.8 \mathrm{mmol} \cdot \mathrm{L}^{-1}\right)$, when values were equal 
to or greater than $190 \mathrm{mg} \cdot \mathrm{dL}^{-1}\left(10.6 \mathrm{mmol} \cdot \mathrm{L}^{-1}\right)$ at $1 \mathrm{~h}$, equal to or greater than $165 \mathrm{mg} \cdot \mathrm{dL}^{-1}\left(9.2 \mathrm{mmol} \cdot \mathrm{L}^{-1}\right)$ at $2 \mathrm{~h}$, or equal to or greater than $145 \mathrm{mg} \cdot \mathrm{dL}^{-1}\left(8.1 \mathrm{mmol} \cdot \mathrm{L}^{-1}\right)$ at $3 \mathrm{~h}$. Diagnosis of GDM occurred if there were at least two abnormal results in the OGTT.

Other pregnancy outcomes. Excessive maternal weight gain was defined according to prepregnancy body mass index (BMI) (20). If prepregnancy BMI was below $18.5 \mathrm{~kg} \cdot \mathrm{m}^{-2}$, the healthy weight gain range was between 12.5 and $18 \mathrm{~kg}$; if BMI was between 18.5 and $24.9 \mathrm{~kg} \cdot \mathrm{m}^{-2}$, the recommendations were between 11.5 and $16 \mathrm{~kg}$; if BMI was between 25 and $29.9 \mathrm{~kg} \cdot \mathrm{m}^{-2}$, healthy weight gain recommendations were between 7 and $11.5 \mathrm{~kg}$; and if BMI was greater than $30 \mathrm{~kg} \cdot \mathrm{m}^{-2}$, weight gain should be between 5 and $9 \mathrm{~kg}(20)$. Weight gain data were taken from medical records.

Gestational age at delivery, type of delivery, birthweight, and length were recorded from medical records. Birthweight less than $2500 \mathrm{~g}$ was considered a low-birthweight baby, and birthweight above $4000 \mathrm{~g}$ was defined as macrosomic (7).

Maternal characteristics. Maternal characteristics recorded from medical records and an initial interview were as follows: age, prepregnant BMI, family history of diabetes, previous GDM, parity, previous miscarriages, history of low birthweight, history of preterm delivery, smoking habits in pregnancy, occupational activity, hours standing, and exercise habits before gestation.

\section{Statistical Analyses}

Statistical analyses were performed with the Statistical Package for Social Sciences software (20.0 version for Mac, Chicago, IL). Data are presented as mean $\pm \mathrm{SD}$ or $n \%$ accordingly. Student's $t$-test was used to compare the means in independent samples (age, prepregnant BMI, 50-g MGS, the OGTT, gestational age, birthweight, and length of newborn). Different variances were assumed when Levene's statistic was less than 0.05 . Pearson $\chi^{2}$ was used for categorical variables (family history of diabetes, previous GDM in pregnancy, parity, previous miscarriages, history of low birthweight, history of preterm delivery, smoking habits in pregnancy, occupational activity, hours standing, exercise habits before gestation, altered 50-g MGS, cases of GDM, excessive weight gain according to prepregnancy BMI, type of delivery, and appropriate weight at birth), concentrating on the probability in $P<0.05$. In the analysis of the categorical tests, Haberman's adjusted residuals were used to interpret with precision the detected association. Logistic regression procedures were used to assess risk estimation (odds ratio (OR)) of GDM in relation to IG and CG. To test the hypothesis of normality, the Kolmogorov-Smirnov test was used.

\section{RESULTS}

From a total of 532 pregnant women interviewed, 342 healthy pregnant women gave written informed consent in

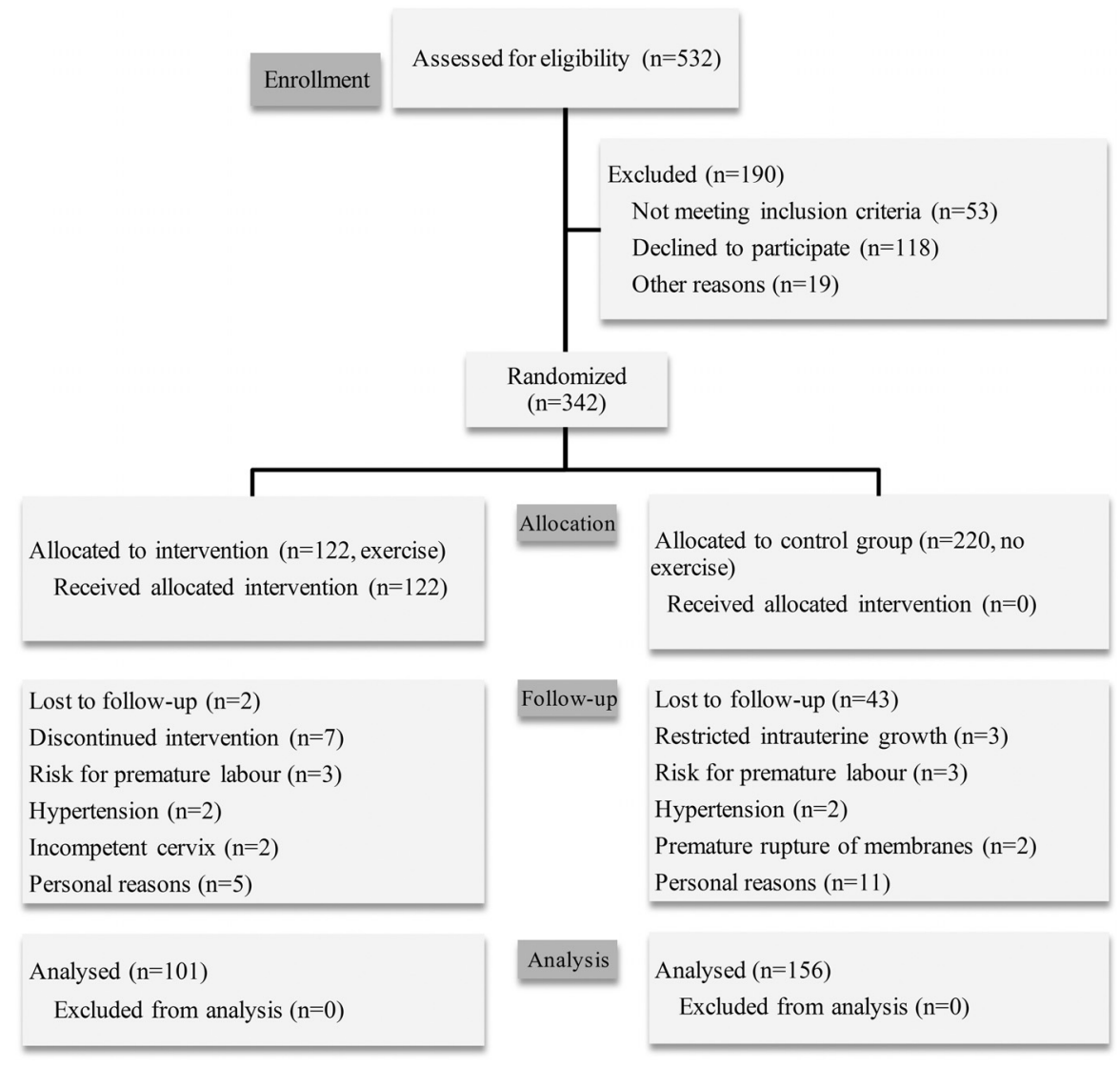

FIGURE 1-Flow diagram of study participants. 
TABLE 1. Maternal characteristics for the IG and the CG.

\begin{tabular}{|c|c|c|}
\hline & IG $(n=101)$ & CG $(n=156)$ \\
\hline Age (yr) & $33.6 \pm 4.1$ & $32.9 \pm 4.5$ \\
\hline Prepregnant BMI $\left(\mathrm{kg} \cdot \mathrm{m}^{-2}\right)$ & $22.5 \pm 3.2$ & $23.6 \pm 4$ \\
\hline \multicolumn{3}{|c|}{ Family history of diabetes $(n \%)$} \\
\hline No & $63 / 62.4$ & $102 / 65.4$ \\
\hline First degree & $23 / 22.8$ & $22 / 14.1$ \\
\hline Second degree & $15 / 14.9$ & $32 / 20.5$ \\
\hline \multicolumn{3}{|c|}{ Previous GDM in pregnancy $(n \%)$} \\
\hline No & $36 / 97.3$ & $80 / 95.2$ \\
\hline Yes & $1 / 2.7$ & $4 / 4.8$ \\
\hline \multicolumn{3}{|l|}{ Parity $(n \%)$} \\
\hline 0 gestation before & $63 / 62.4$ & $74 / 47.4$ \\
\hline 1 gestation before & $30 / 29.7$ & $62 / 39.7$ \\
\hline$>1$ gestation before & $8 / 7.9$ & $20 / 12.8$ \\
\hline \multicolumn{3}{|l|}{ Previous miscarriages ( $n \%$ ) } \\
\hline 0 & $69 / 68.3$ & $105 / 67.3$ \\
\hline 1 & $25 / 24.8$ & $40 / 25.6$ \\
\hline$>1$ & $7 / 6.9$ & $11 / 7.1$ \\
\hline \multicolumn{3}{|c|}{ History of low birthweight $(n \%)$} \\
\hline 0 & $97 / 96$ & $147 / 94.2$ \\
\hline 1 & $3 / 3$ & $8 / 5.1$ \\
\hline$>1$ & $1 / 1$ & $1 / 0.6$ \\
\hline \multicolumn{3}{|c|}{ History of preterm delivery $(n \%)$} \\
\hline 0 & $101 / 100$ & $152 / 97.4$ \\
\hline 1 & $0 / 0$ & $4 / 2.6$ \\
\hline \multicolumn{3}{|c|}{ Smoking habits in pregnancy $(n \%)$} \\
\hline No & $98 / 97$ & $147 / 94.2$ \\
\hline Yes & $3 / 3$ & $9 / 5.8$ \\
\hline \multicolumn{3}{|l|}{ Occupational activity $(n \%)$} \\
\hline Sedentary job & $65 / 64.4$ & $92 / 59$ \\
\hline Housewife & $6 / 5.9$ & $11 / 7.1$ \\
\hline Active job & $30 / 29.7$ & $53 / 34$ \\
\hline \multicolumn{3}{|l|}{ Hours standing $(n \%)$} \\
\hline$\leq 3 \mathrm{~h}$ & $48 / 47.5$ & $71 / 45.5$ \\
\hline$>3 \mathrm{~h}$ & $53 / 52.5$ & $85 / 54.5$ \\
\hline \multicolumn{3}{|c|}{ Exercise habits before gestation $(n \%)$} \\
\hline Sedentary & $18 / 17.8$ & $43 / 27.6$ \\
\hline Active & $83 / 82.2$ & $113 / 72.4$ \\
\hline
\end{tabular}

Data are expressed as mean \pm SD, unless otherwise indicated.

agreement with the last modification of the Declaration of Helsinki (36), 122 of whom were randomized to the IG and 220 were randomized to the CG.

During the course of the study, 21 pregnant women from the IG were excluded (16.4\%) because of the following: lost to follow-up $(n=2)$, discontinued intervention $(n=7)$, risk for premature labor $(n=3)$, hypertension $(n=2)$, incompetent cervix $(n=2)$, and personal reasons $(n=5)$. Sixty-four women from the CG were excluded $(29.1 \%)$ because of the following: lost to follow-up $(n=43)$, restricted intrauterine growth $(n=3)$, risk for premature labor $(n=3)$, hypertension $(n=2)$, premature rupture of membranes $(n=2)$, and personal reasons $(n=11)$. The final number of pregnant women analyzed was 257 (Fig. 1).

In Table 1, maternal characteristics at the beginning of the study are presented. No significant differences between groups were found.

There were no significant differences for the 50-g MGS values between the groups; however, the glucose values corresponding to the OGTT at $180 \mathrm{~min}$ showed that the IG had lower values (IG, $98.00 \pm 29.48 \mathrm{mg} \cdot \mathrm{dL}^{-1}$, vs $\mathrm{CG}, 116.25 \pm$ $\left.29.90 \mathrm{mg} \cdot \mathrm{dL}^{-1}\left(t_{64}=2.37, P=0.021\right)\right)$. There was a significant difference between the number of cases diagnosed with GDM between the two groups (IG, $1 \%, n=1$, vs CG, $8.8 \%$, $\left.n=13\left(\chi_{1}^{2}=6.84, P=0.009\right)\right)$. Women who engaged exercise program during pregnancy compared with $\mathrm{CG}$ experienced a $90 \%$ reduced risk of GDM (OR $=0.103 ; 95 \%$ confidence interval (CI), 0.01-0.803) (Table 2).

Significant differences were found between groups in excessive maternal weight gain according to prepregnancy BMI classes (IG, $22.8 \%, n=23$, vs CG, $34.8 \%, n=54\left(\chi_{1}^{2}=4.23\right.$, $P=0.04)$ ). No other significant differences were found in any other pregnancy outcomes (Table 3).

\section{DISCUSSION}

Maternal exercise using both aerobic and muscular conditioning on land and in the water with high compliance reduced the incidence of GDM, is strongly associated with a decrease in gestational weight gain, and preserved glucose tolerance (Table 2).

There is controversy that physical exercise during pregnancy prevents GDM. Callaway et al. (5), with a sample of 25 subjects per arm and an individualized exercise program via e-mail and telephone support from week 12 in obese women, found improvement in glucose tolerance. Dye et al. (15) used the same statistical treatment as the present study, with contingency tables and Pearson $\chi^{2}$ to examine differences in prevalence, and found reduced rates of GDM in pregnant women with a BMI greater than $33 \mathrm{~kg} \cdot \mathrm{m}^{-2}$. Physical exercise was measured through telephone interviews. Dempsey et al. $(12,13)$ reported that women engaging in some sort of recreational physical activity during pregnancy reduced the risk of GDM. They emphasized physical activity during early

TABLE 2. Glucose values for the 50-g MGS (the fasted OGTT and the number of cases diagnosed with GDM for the IG and the CG).

\begin{tabular}{|c|c|c|c|c|c|}
\hline & & IG $(n=100)$ & CG $(n=146)$ & $P$ Value & OR (95\% CI) \\
\hline 50-g MGS & $\begin{array}{l}\left(\mathrm{mg} \cdot \mathrm{dL}^{-1}\right) \\
\left(\mathrm{mmol} \cdot \mathrm{L}^{-1}\right)\end{array}$ & $\begin{aligned} 116.72 & \pm 27.79 \\
6.5 & \pm 1.5\end{aligned}$ & $\begin{array}{c}123.66 \pm 32.23 \\
6.9 \pm 1.8\end{array}$ & 0.085 & \\
\hline Altered $50-\mathrm{g}$ MGS $(n \%)$ & & $23 / 23$ & $43 / 29.5$ & 0.262 & \\
\hline Fasted & $\begin{array}{l}\left(\mathrm{mg} \cdot \mathrm{dL}^{-1}\right) \\
\left(\mathrm{mmol} \cdot \mathrm{L}^{-1}\right)\end{array}$ & $\begin{aligned} 79.95 & \pm 10.74 \\
4.4 & \pm 0.6\end{aligned}$ & $\begin{aligned} 84.71 & \pm 8 \\
4.7 & \pm 0.4\end{aligned}$ & 0.051 & \\
\hline 60-min 0GTT & $\begin{array}{l}\left(\mathrm{mg} \cdot \mathrm{dL}^{-1}\right) \\
\left(\mathrm{mmol} \cdot \mathrm{L}^{-1}\right)\end{array}$ & $\begin{aligned} 155.30 & \pm 27.16 \\
8.6 & \pm 1.5\end{aligned}$ & $\begin{array}{c}160.33 \pm 29.53 \\
8.9 \pm 1.6\end{array}$ & 0.502 & \\
\hline 120-min OGTT & $\begin{array}{l}\left(\mathrm{mg} \cdot \mathrm{dL}^{-1}\right) \\
\left(\mathrm{mmol} \cdot \mathrm{L}^{-1}\right)\end{array}$ & $\begin{aligned} 124.87 & \pm 27.71 \\
6.9 & \pm 1.5\end{aligned}$ & $\begin{array}{c}138.54 \pm 33.07 \\
7.7 \pm 1.8\end{array}$ & 0.097 & \\
\hline 180-min OGTT & $\begin{array}{l}\left(\mathrm{mg} \cdot \mathrm{dL}^{-1}\right) \\
\left(\mathrm{mmol} \cdot \mathrm{L}^{-1}\right)\end{array}$ & $\begin{aligned} 98.00 & \pm 29.48 \\
5.4 & \pm 1.6\end{aligned}$ & $\begin{array}{c}116.25 \pm 29.90 \\
6.5 \pm 1.7\end{array}$ & $0.021^{*}$ & \\
\hline Cases of GDM $(n \%)$ & & $1 / 1$ & $13 / 8.9$ & $0.009^{*}$ & $0.103(0.013-0.803)$ \\
\hline
\end{tabular}

Data are expressed as mean $\pm \mathrm{SD}$, unless otherwise indicated.

Glucose data were not available for 11 women.

${ }^{*} P<0.05$. 


\begin{tabular}{|c|c|c|c|}
\hline & IG $(n=101)$ & CG $(n=156)$ & $P$ Value \\
\hline Excessive weight gain according to prepregnancy $\mathrm{BMI}(n \%)$ & & & $0.040^{*}$ \\
\hline No & $78 / 77.2$ & $101 / 65.2$ & \\
\hline Yes & $23 / 22.8$ & $54 / 34.8$ & \\
\hline Gestational age $(\mathrm{d})$ & $277.69 \pm 8.45$ & $276.74 \pm 10.58$ & 0.446 \\
\hline Type of delivery $(n \%)$ & & & 0.626 \\
\hline Normal & $60 / 59.4$ & $95 / 60.9$ & \\
\hline Instrumental & $15 / 14.9$ & $28 / 17.9$ & \\
\hline Caesarean & $26 / 25.7$ & $33 / 21.2$ & \\
\hline \multicolumn{4}{|l|}{ Newborn outcome } \\
\hline Birthweight $(\mathrm{g})$ & $3324.1 \pm 433.1$ & $3250.1 \pm 425.01$ & 0.177 \\
\hline Appropriate weight $(n \%)$ & & & 0.579 \\
\hline No (below $2500 \mathrm{~g}$ ) & $3 / 3$ & $9 / 5.8$ & \\
\hline Yes & $93 / 92.1$ & $140 / 89.7$ & \\
\hline No (above $4000 \mathrm{~g}$ ) & $5 / 5$ & $7 / 4.5$ & \\
\hline Length of newborn (cm) & $49.94 \pm 2.16$ & $49.56 \pm 1.99$ & 0.151 \\
\hline
\end{tabular}

Data are expressed as mean $\pm \mathrm{SD}$, unless otherwise indicated.

Excessive weight gain data were not available for one woman in the CG; length of newborn data were not available for three newborns (one in IG and two in CG). ${ }^{\star} P<0.05$.

gestation. Liu et al. (22) reported that physical activity reduced the prevalence of GDM in women. Both studies were observational and information was collected through surveys (12,22). Stafne et al. (33) conducted a similar study, with moderate- to vigorous-intensity exercise in healthy pregnant women independent of BMI, but their trial started later in pregnancy at 18-22 gestational weeks and no differences were reported between the groups. It may be that early exercise intervention may be key to GDM prevention.

In the present study, exercise also reduced excessive maternal weight gain. Avoiding excessive weight gain during pregnancy can prevent excessive weight retention after birth $(6,16,20)$. There is increasing evidence that reports better control of glucose metabolism in pregnant women who exercise compared to those who remain sedentary, both in healthy pregnant women and in pregnant women diagnosed with $\operatorname{GDM}(5,10,11,18,24,29)$. Furthermore, increase lipid blood levels in pregnancy due to excessive gestational weight gain may be linked to insulin resistance $(6,8)$ and may be a factor in developing GDM (25).

Weight gain and obesity promote type 2 diabetes in nonpregnant women (21). In the gestational period, the literature focuses on and reports the existence of predisposition to diabetes in obese women $(25,35)$ rather than the independence of the prepregnancy BMI category. Also, in recent years, a relationship has been found between excessive weight gain according to the Institute of Medicine BMI classification for weight gain recommendations and glucose intolerance without actually resulting in the pathology (19). Our study fails to statistically confirm that a causal relationship between both variables exists, but the literature seems to indicate so. In an epidemiological study, weight gain during pregnancy and the incidence of GDM were shown to be causally related (31).

Many studies evaluating the role of physical exercise on pregnancy outcome and the prevention of GDM used different intervention protocols such as the provision of several prenatal visits or contact via email/telephone $(5,15)$, or observational studies in which information about the physical activity performed by the mother is collected through interviews/questionnaires $(12,15,22)$.
There are only a few studies in which all program sessions take place in groups and with the supervision of obstetricians and those qualified in Exercise Science for the guidance of the program. In our opinion, the fact that we offered a dynamic, controlled supervised program, with a variety of options in the sessions, and using the dynamics of a group, resulted in a high level of adherence and, therefore, may explain the effectiveness of our protocol. Compliance may be an important issue when evaluating the efficacy and effectiveness of any randomized controlled trial, especially relating to an intervention during pregnancy. The main limitation of the present study was that there was no nutrition analyses but all women were exposed to the same standard care that emphasizes healthy eating and a healthy lifestyle. The only difference between the groups was the exercise program.

Overall, physical inactivity is associated with the risk of glucose intolerance (18), and physical activity is associated with better response to glucose metabolism (11), but there is a lack of specific recommendations regarding frequency and type of exercise, when to start, the duration, and the intensity of the exercise sessions that can provide effective results for the prevention of the disease (24). Based on our results, to reduce the incidence of GDM, a frequency of at least three times per week, with a mixture of aerobic and muscle conditioning exercise, on land and in water, with sessions lasting at least $50 \mathrm{~min}$ with $10-\mathrm{min}$ warm-up and $10 \mathrm{~min}$ cool down, with an intensity of $60 \%$ predicted HR reserve (12-14; somewhat strong on Borg's scale), may be suggested.

The authors thank the Technical University of Madrid and the Gynecology and Obstetric Department of the following center which belong to Area 6 of the autonomous community of Madrid: the Puerta de Hierro University Hospital, the Torrelodones University Hospital, and the Torrelodones Health Center. The authors also thank Torrelodones Municipal Pool where the aquatic activities were developed and all the pregnant women who participated in this study.

The study was partially supported by the Ministerio de Ciencia e Innovación, Spain (DEP2009-08990). No conflicts of interest relevant to this article were reported.

This study was registered at www.clinicaltrials.gov, identifier: NCT01790412.

The results of the present study do not constitute endorsement by the American College of Sports Medicine. 


\section{REFERENCES}

1. American College of Obstetricians and Gynecologists. Exercise during pregnancy and the postpartum period. Obstet Gynecol. 2002; 99:171-3.

2. Artal R, Toole MO. Guidelines of the American College of Obstetricians and Gynecologists for exercise during pregnancy and the postpartum period. Br J Sports Med. 2003;37:6-12.

3. Bellamy L, Casas JP, Hingorani AD, Williams D. Type 2 diabetes mellitus after gestational diabetes: a systematic review and metaanalysis. Lancet. 2009;23(373 (9677)):1773-9.

4. Borg GA. Psychophysical bases of perceived exertion. Med Sci Sports Exerc. 1982;14(5):377-81.

5. Callaway LK, Colditz PB, Byrne NM, et al. Prevention of gestational diabetes: feasibility issues for an exercise intervention in obese pregnant women. Diabetes Care. 2010;33(7):1457-9.

6. Catalano PM. Obesity, insulin resistance, and pregnancy outcome. Reproduction. 2010;140(3):365-71.

7. Chen X, Scholl T. Gestational hyperglycemia, excessive pregnancy weight gain and risk of fetal overgrowth. In: Radenkovic M, editor. Gestational Diabetes. Croatia: InTech; 2011: pp. 243-54.

8. Chyad Al-Noaemi M, Helmy Faris Shalayel M. Pathophysiology of gestational diabetes mellitus: the past, the present and the future. In: Radenkovic M, editor. Gestational Diabetes. Croatia: InTech; 2011. pp. 91-114.

9. Cohen J. Quantitative methods in psychology. A power primer. Psychol Bull. 1992;112(1):155-9.

10. de Barros MC, Lopes MA, Francisco RP, Sapienza AD, Zugaib M. Resistance exercise and glycemic control in women with gestational diabetes mellitus. Am J Obstet Gynecol. 2010;203(6):556.

11. Deierlein AL, Siega-Riz AM, Evenson KR. Physical activity during pregnancy and risk of hyperglycemia. $J$ Womens Health (Larchmt). 2012;21(7):769-75.

12. Dempsey JC, Butler CL, Sorensen TK, et al. A case-control study of maternal recreational physical activity and risk of gestational diabetes mellitus. Diabetes Res Clin Pract. 2004;66(2):203-15.

13. Dempsey JC, Sorensen TK, Williams MA, et al. Prospective study of gestational diabetes mellitus risk in relation to maternal recreational physical activity before and during pregnancy. Am J Epidemiol. 2004; 159(7):663-70.

14. Downs DS, Chasan-Taber L, Evenson KR, Leiferman J, Yeo S. Physical activity and pregnancy: past and present evidence and future recommendations. Res Q Exerc Sport. 2012;83(4):485-502.

15. Dye TD, Knox KL, Artal R, Aubry RH, Wojtowycz MA. Physical activity, obesity, and diabetes in pregnancy. Am J Epidemiol. 1997;146(11):961-5.

16. Ferrara A, Hedderson MM, Albright CL, et al. A pregnancy and postpartum lifestyle intervention in women with gestational diabetes mellitus reduces diabetes risk factors: a feasibility randomized control trial. Diabetes Care. 2011;34(7):1519-25.

17. Goldberg L, Elliot DL, Kuehl KS. Assessment of exercise intensity formulas by use of ventilatory threshold. Chest. 1988;94(1):95-8.

18. Gollenberg AL, Pekow P, Bertone-Johnson ER, Freedson PS, Markenson G, Chasan-Taber L. Sedentary behaviors and abnormal glucose tolerance among pregnant Latina women. Med Sci Sports Exerc. 2010;42(6):1079-85.

19. Herring SJ, Oken E, Rifas-Shiman SL, et al. Weight gain in pregnancy and risk of maternal hyperglycemia. Am J Obstet Gynecol. 2009;201(1):61.e1-7.

20. Institute of Medicine. Institute of Medicine and National Research Council. In: Rasmussen KM, Yaktine AL, editors. Weight Gain
During Pregnancy: Reexamining the Guidelines. Washington (DC): National Academy of Sciences; 2009.

21. Krotkiewski M, Seidell JC, Bjorntorp P. Glucose tolerance and hyperinsulinaemia in obese women: role of adipose tissue distribution, muscle fibre characteristics and androgens. J Intern Med. 1990;228(4):385-92.

22. Liu J, Laditka JN, Mayer-Davis EJ, Pate RR. Does physical activity during pregnancy reduce the risk of gestational diabetes among previously inactive women? Birth. 2008;35(3):188-95.

23. Liu JH, Mayer-Davis EJ, Pate RR, Gallagher AE, Bacon JL. Physical activity during pregnancy is associated with reduced fasting insulin - the Pilot Pregnancy and Active Living Study. J Matern Fetal Neonatal Med. 2010;23(10):1249-52.

24. Mottola MF. The role of exercise in the prevention and treatment of gestational diabetes mellitus. Curr Diab Rep. 2008;8(4):299-304.

25. Mottola MF, Ruchat SM. Exercise guidelines for women with gestational diabetes. In: Radenkovic M, editor. Gestational Diabetes. Croatia: InTech; 2011: pp. 339-62.

26. Oostdam N, van Poppel MN, Wouters MG, van Mechelen W. Interventions for preventing gestational diabetes mellitus: a systematic review and meta-analysis. $J$ Womens Health (Larchmt). 2011;20(10):1551-63.

27. Petkova V, Nikolova I. Prevalence and risk factors for the development of GD in some eastern European countries - tendencies and pharmacoeconomical assessment for the choice of treatment. In: Radenkovic M, editor. Gestational Diabetes. Croatia: InTech, 2011: pp. 69-90.

28. Ricart W, Lopez J, Mozas J, et al. Potential impact of American Diabetes Association (2000) criteria for diagnosis of gestational diabetes mellitus in Spain. Diabetologia. 2005;48(6):1135-341.

29. Ruchat SM, Davenport MH, Giroux I, et al. Effect of exercise intensity and duration on capillary glucose responses in pregnant women at low and high risk for gestational diabetes. Diabetes Metab Res Rev. 2012;28(8):669-78.

30. Seshiah V, Balaji V, Madhuri B. Gestational diabetes mellitus-a perspective. In: Radenkovic M, editor. Gestational Diabetes. Croatia: InTech; 2011: pp. 21-40.

31. Sommer C, Morkrid K, Jenum AK, Sletner L, Mosdol A, Birkeland KI. Weight gain, total fat gain and regional fat gain during pregnancy and the association with gestational diabetes: a populationbased cohort study. Int J Obes. 2013;38(1):76-81.

32. Soultanakis NH, Artal R, Wiswell RA. Prolonged exercise in pregnancy: glucose homeostasis, ventilatory and cardiovascular responses. Semin Perinatol. 1996;20(4):315-27.

33. Stafne SN, Salvesen KA, Romundstad PR, Eggebo TM, Carlsen SM, Morkved S. Regular exercise during pregnancy to prevent gestational diabetes: a randomized controlled trial. Obstet Gynecol. 2012;119(1):29-36.

34. Tobias DK, Zhang C, van Dam RM, Bowers K, Hu FB. Physical activity before and during pregnancy and risk of gestational diabetes mellitus: a meta-analysis. Diabetes Care. 2011;34(1):223-9.

35. Tovar A, Must A, Bermudez OI, Hyatt RR, Chasan-Taber L. The impact of gestational weight gain and diet on abnormal glucose tolerance during pregnancy in Hispanic women. Matern Child Health J. 2009;13(4):520-30.

36. Williams JR. The Declaration of Helsinki and public health. Bull World Health Organ. 2008;86(8):650-2.

37. Zorzano A, Munoz P, Camps M, Mora C, Testar X, Palacin M. Insulin-induced redistribution of GLUT4 glucose carriers in the muscle fiber. In search of GLUT4 trafficking pathways. Diabetes. 1996;45(1 Suppl):S70-81. 